\title{
ERGO-MTM model: an integrated approach to set working times based upon standardized working performance and controlled biomechanical load
}

\author{
Caragnano G. ${ }^{\mathrm{a} *}$, Lavatelli I. ${ }^{\mathrm{a}}$ \\ ${ }^{a}$ PricewaterhouseCoopers Advisory SpA, Operations, Milan, Italy
}

\begin{abstract}
ERGO-MTM is an innovative model to set standard times of manual tasks. MTM, developed in the forties by industrial engineers, assigns a basic time to execute a given motion based on the concept of normal performance (speed, effort and precision). The traditional models to set a standard time then add to the basic time a fatigue allowance, applied on each individual motion, depending generally on the type of posture and on the load/force implied in the motion. To the light of the most recent ISO/CEN standards dealing with biomechanical load, the traditional models do not meet the requirements any more: it becomes mandatory to consider the load generated by the overall assignment of working tasks to a workstation to be compliant with the new ergonomics standards. ERGO-MTM is the solution: it determines a fatigue allowance (named Ergonomic Allowance), which is applied on the total workstation basic MTM time to allow the necessary recovery periods, enough to keep the biomechanical load within safety limits. The final result is a standard time based on a norm level of performance and a work sequence with a controlled biomechanical load.
\end{abstract}

Keywords: ERGO-MTM, EAWS, performance, recovery allowance, standard time

\section{Work measurement and task assignment require the use of predetermined time systems and the biomechanical load control to design and implement efficient and safe working systems}

Work measurement is definitively a difficult task, since we miss a clear and objective reference as for distances and weights, where we have the meter and the kilogram. To set a standard time of a given manual task the following steps are necessary:

- Set a basic time

- Determine the proper time allowances

- Add allowances to the basic time to set a standard time

A basic time is the time necessary to an average person, well instructed, moving at an average speed and paying an average effort to accomplish a task, under the hypothesis to work for an entire shift (ap- prox. 8 hours). These conditions (skills, speed and effort) merge into a factor named working performance. When setting a basic time of a task with a time study, the analyst has to level the observed time using the normal performance as a reference (if the rated performance is higher than the normal performance, then the basic time would be longer than the observed time). For example, to calculate the basic time to walk $1 \mathrm{~km}$, it's necessary to observe a person walking $1 \mathrm{~km}$, rate the performance and take his/her actual time to execute the task. Imagine that the stop-watch time is $800 \mathrm{~s}$ and that the rated performance is $110 \%$ ( $10 \%$ above the one of an average person walking without getting short of breath). The standard time to walk $1 \mathrm{~km}$ then would be $800 \mathrm{~s} \mathrm{x} 1.1=880 \mathrm{~s}$. If a person takes $880 \mathrm{~s}$ to walk $1 \mathrm{~km}$, his/her performance would be exactly $100 \%$.

*Corresponding author: E-mail: Gabriele.caragnano@it.pwc.com. 
The use of a predetermined time system like MTM (Methods-Time Measurement) makes the performance rating unnecessary, eliminating a large degree of subjectivity, since all the basic motions (e.g. reach, grasp, move, etc.) have been measured and are by definition built upon the normal level of performance (named MTM normal performance). At present the MTM normal performance is the most used and best known performance reference in the World.

Unfortunately there still exist today work measurement scales which position the normal work pace at a level which is even $20 \%$ higher than the MTM normal performance. For example, the ILO standard performance is defined as "the rate of output which qualified workers will naturally achieve without over exertion as an average over the working day or shift, provided that they are motivated to apply themselves to their work.

Each of the rating systems/scales [1] starts from a different conceptual viewpoint. The Bedaux System (now little used) assumed that 'normal' performance was 60 'minutes of work' per hour, that 80 'minutes of work' per hour was incentive performance and that 100 was a theoretical maximum.

One of the common problems of rating is that it is often linked to remuneration, through the setting of 'daywork' rates or through graduated incentive payment schemes. This results in pressure from employees and unions on work study practitioners to 'slacken' their ratings to give 'looser' time values for jobs.

Some attempts have been made to compare results derived from the use of different systems/scales. The following information is just a collection from various sources and it is not to be considered officially recognized [2]:

British Standard BS3138 gives a comparison of the BSI scale with the Bedaux Scale which gives BSI = Bedaux x 1.25.

Another often quoted figure for the BSI rating scale is that MTM $100=83$ on the BSI scale.

There is no empirical evidence for this conversion, but it is in general usage and we can thus summarise this combined data as below.

\begin{tabular}{ccc}
\hline MTM & BSI & BEDAUX \\
\hline 95,2 & 79,0 & 63,2 \\
\hline 100,0 & 83,0 & 66,4 \\
\hline 105,7 & 87,7 & 70,2 \\
\hline 120,5 & 100,0 & 80,0 \\
\hline 150,6 & 125,0 & 100,0 \\
\hline
\end{tabular}

Figure 1 - comparison of performance rating scales

MTM (normal performance $=100$ ) and Bedaux (normal performance $=80$ ) are classified as 'Medium Task Systems' (they are all within $\pm 5 \%$ with respect to MTM)

MTM normal performance is recognized worldwide and it is considered as a fair level for setting basic times (it's neither low nor high level). In numerous MTM application experiences, typical actual performance levels, measured on the MTM scale by qualified international experts, range from $80 \%$ to $120 \%$ with a confidence level of $95 \%$.

BSI scale is a 'High Task System' (+ 21\% with respect to MTM).

From recent studies conducted to develop the ergonomic screening system 'European Assembly Work-Sheet' (EAWS), the MTM normal performance results to be in line with the most recent ergonomic standards related to the biomechanical load.

That further confirms the concept of medium performance as opposed to high.

The allowances are thought to compensate for fatigue and delays at work. Industrial Engineering (IE) practice distinguishes between constant and variable allowances: the former cover the personal needs (e.g. going to the restroom) and the basic fatigue, the latter deals with body postures, forces, loads and highfrequency movements of the upper limbs (fatigue allowance). Fatigue allowances have not reached the state where their qualifications are completely based on sound, rational theories. Consequently, next to performance rating, the fatigue allowance is the least defensible and the most open to argument of all the factors making up a time standard.

Although heavy manual work, and thus muscular fatigue, is diminishing in industry, due to mechanization, other fatigue components, such as mental stress and monotony, may be increasing. Because all fatigue cannot be eliminated, proper allowance must be made for the working conditions and repetitiveness of the work. 
The last step, once allowances have been set, is to add them to the basic time as a percentage of the basic time.

\section{The ERGO-MTM model to set standard times}

The ERGO-MTM model is the result of the integration of 2 systems:

- MTM to clearly identify the necessary working motions and conditions to accomplish a task and calculate the basic time

- EAWS (European Assembly Work-Sheet) to calculate the biomechanical load level of the same task

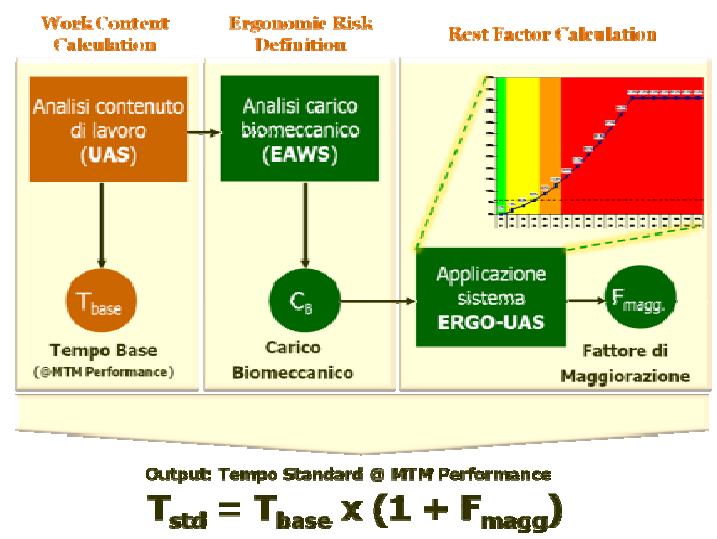

Figure 2-ERGO_MTM calculation steps

EAWS is a first level risk assessment method for the biomechanical load. It was designed and developed to be an holistic system considering the main relevant biomechanical influencing factors: the body postures, the forces, the loads and the repetitive motions of the upper limbs.

The ERGO-MTM model, in its conceptual simplicity, is quite innovative, since it introduces a further dimension to assess the fatigue allowance: the Time factor. Indeed, the traditional methods (like the ILO recommended table of allowances) based the determination of the allowance as a function of the main body posture and of the force/load level, regardless the duration or the frequency of the motions. The allowance is then applied on each single motion, and for this reason the model is called "single-motion allowance" model. For example, to lift a load of 40 pounds, the ILO Recommended Allowance table [3] gives a value of $9 \%$. No matter if that action was repeated once or 10 times per cycle!

The ERGO-MTM model assigns an Ergonomic allowance (fatigue allowance) as a function of the EAWS score, which is an index tightly linked to the concept of physical workload.

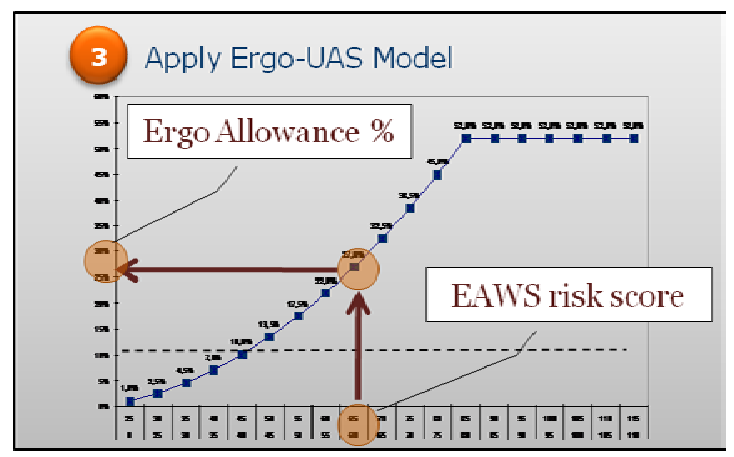

Figure 3 - ERGO-MTM model

In EAWS the phisical workload is calculated as follows:

Work-load $=$ Intensity $\mathrm{x}$ Time

Intensity is proportional to the awkwardness of the postures, the force intensity, the load weight etc. [4]

Time depends on the duration of the static actions and the frequency of the dynamic actions.

To get to a significant level of work-load, it's necessary to reach at least a medium intensity with a medium time factor. If either one of the two factors is negligible, the resulting work-load would be low, even if the other factor is high.

The main advantages of the ERGO-MTM model are:

- Link with relevant ISO/CEN standards in measuring the phisical load (mainly CEN 1005 and ISO 11226, 11228)

- Objective motions identification through the link with the MTM language (easy to identify and count actions)

- Normalized motion frequency calculation based on MTM basic times (action frequencies are not influenced by the operator actual performance)

- Data consistency granted by the link with work cycles (if a work method or a piece of equipment is modified, than the workload is automatically updated) 
- Integration of two job profiles (method engineer and ergonomist) into one (ergo-engineer) with ensuing cost savings

- Focus on work method as a means to improve productivity and ergonomic conditions

- Easier to justify investems in ergonomic improvement projects, since product cost is linked with the phisical workload level (higher load results in higher fatigue allowance)

\section{Prototyping the ERGO-MTM model}

Given that in a normal industrial organization the total amounts of pauses ranges between 30 and 60 minutes, it can be stated that constant allowances are absorbed by those structural breaks. Variable allowances instead should be set with the objective to keep the work-load under control.

Physical work [5] is peformed by the activation of the musculoskeletal system. The muscular contractions during work require the support of the respiratory and circulatory systems in order to transport oxygen to, and to carry metabolic byproducts from, the muscles. Consequently, the responses of these supporting systems are closely correlated to the intensity of the work. In engineering terms the work can be considered the stress, and the physiological responses the ensuing strain. Therefore the physiological responses can be used to estimate performance and to design the work.

The nature of the physiological strain depends on the type of muscular contraction involved. There are two types of muscular contractions:

- Dynamic, involving rythmical contractions of large muscle groups where the length of the muscles is changing (isotonic)

- Static, involving prolonged contraction without a change in the length of the muscles (isometric)

The ERGO-MTM model was built upon the fundamental principle to allow a quantity of recovery time enough to keep the physical load within controlled limits. Two ways were followed to set those limits: the former is to crosscheck the allowances against recovery periods calculated with a formula defined by the German school of occupational physiologists [5]:

$$
R A=\left(\frac{M}{42}-1\right) \times 100
$$

Where RA is rest allowance as percentage of the working time (MTM basic time), $\mathrm{M}$ is the metabolic cost of the work in $\mathrm{Kcal} / \mathrm{min}$, and the constant, 4.2, represents the basic cost of work that does not require rest allowance. The $4.2 \mathrm{Kcal} / \mathrm{min}$ was derived from calculated energy requirements of workers whose nutritional need were followed for a prolonged time during World War I and II.

In the following tables $[6,7]$ some energy expenditure values are reported :

\begin{tabular}{lc}
\hline Task & $\begin{array}{c}\text { M } \\
\text { (kcal/min) }\end{array}$ \\
Small item packing & 2.5 \\
Using vacuum cleaner & 3.5 \\
Window washing & 5.0 \\
Wet mop lobby room (1 kg mop) & 6.5 \\
Aluminum smelting - Shovel & 9.0 \\
Steel mill - slag removal & 11.5 \\
Aluminum smelting - Break bath off anode & 15.0 \\
\hline
\end{tabular}

Figure 4: Energy expenditure per typical tasks

Considering for example a well known task like the wet mop lobby room, using the values in Figure 4, it can be calculated that that task requires an energy expenditure approximately $50 \%$ higher than the basic cost value of $4.2 \mathrm{kcal} / \mathrm{min}$, generating, using Eq (1), a $\mathrm{RA}=50 \%$. It means that out of one hour, $40 \mathrm{~min}$ are working time and $20 \mathrm{~min}$ are rest.

The latter is to allow growing values of rest (\% ergonomic allowance), starting from $0 \%$ at 25 EAWS points (green zone, absence of risk), at any increase of biomechanical load (using steps of 5 EAWS points) in a way to match $6 \%$ at the mid of the yellow zone (EAWS score 35-40) and to accelerate up to $51 \%$ at an EAWS score of 80 . The benchmark of $6 \%$ is determined in order to assign a typical average fatigue allowance value used by most of the industries (in particular in the automotive and white goods industries) at medium work load level (30-35 EAWS score). The high end percentage of $51 \%$ was determined using an experimental approach: which are the necessary ergonomic allowances capable to assign enough rest in order to lower the EAWS score below the red zone limit (50 EAWS score). The best fitting 
function is exponential: starting from $0 \%$ at 25 points, it grows assigning incremental percentages at each step of 5 EAWS points:

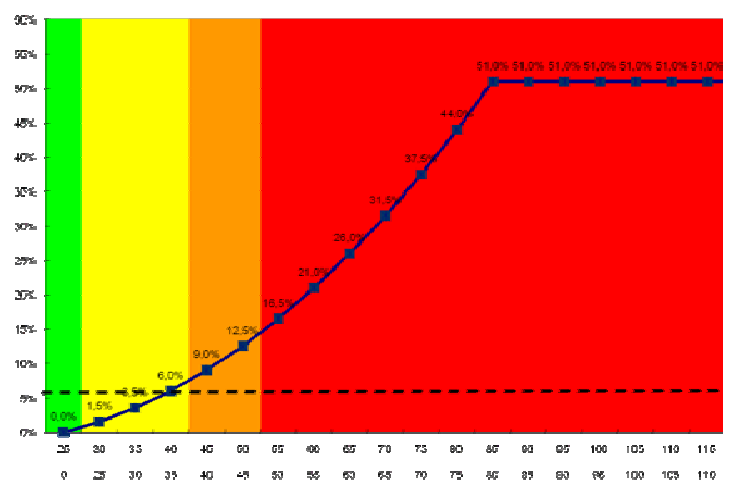

Figure 5: ERGO-MTM curve

The mutual compliance between the two methods was checked through some analyses. For example, if we take the task "Wet mop lobby room (1 kg mop)", in Table 1 it is shown that the metabolic cost is 6.5 $\mathrm{kcal} / \mathrm{min}$. Using Eq (1), the RA results to be $55 \%$.

The task was analyzed with the MTM-UAS system in order to describe in details the method and calculate the standard duration of all movements. The work organization was assumed to have a cycle of 1 minute repeated continuously in a shift of 8 hours. The result of the EAWS calculation [8] was the following:

Table 6

EAWS scores

\begin{tabular}{|c|c|c|c|c|c|c|}
\hline \multicolumn{3}{|c|}{ Whole body } & \multicolumn{4}{|c|}{ Upper limbs } \\
\hline & Body posture & 13,00 Points & & & Task & 4,30 Points \\
\hline & + Forces & 31,00 Points & & $1+$ & Hand/Arm/Shoulde & 0,00 Points \\
\hline 8 & + Loads & 42,50 Points & $|0|$ & + & Further factors & 0,00 Points \\
\hline & \begin{tabular}{l|l}
+ Extra points \\
\end{tabular} & 0,00 Points & 10 & * & Duration & 8,00 Points \\
\hline & Total points & 86,50 Points & & & Total points & 35,00 Points \\
\hline
\end{tabular}

The final score to be used in the ERGO-MTM model is then 86,5 (the highest value between whole body and upper limbs). At that score an ergonomic allowance of $51 \%$ is assigned by the ERGO-MTM model, which is definitively close to the value obtained with the energy consumption equation (55\%).

\section{Conclusions}

To the light of the many evidences and medical data available today, it's quite clear there is a strong correlation between the physical workload and the probability to get sick (WRMSD - Work Related Musculo-Skeletal Disorders). It's a huge responsibility by the industrial engineers to design efficient and safe work systems to grant competitive levels of productivity in full respect of the safety regulations. The ERGO-MTM model forces the ergo-engineer to consider all types of constraints and risks from an early stage of the product and process development. The key ingredients of the model are MTM and EAWS. MTM ensures a detailed motion identification and a fair normal time determination. EAWS is an holistic first level approach to the measurement of the biomechanical load, considering the main load ingredients within the same structure and score (postures, forces, repetitive motions of the upper limbs).

A last but very important note regards the work measurement system: in order to implement a sound and consistent work planning and control process, which covers of course also the ergonomic matters, it's absolutely and extremely important to adopt a reliable and fair work measurement system. It's strongly recommended to select a predetermined time system (objective, preventive and method oriented) based on a performance scale which fits the relevant ISO/CEN standards. The ERGO-MTM model is built upon the MTM performance level, which is considered as a fair level by most of the Unions and Industries around the globe.

The International MTM Directorate (IMD) recommends to adopt a medium task scale to define a fair performance level. MTM does not exploit workers and, if used in combination with a physical load screening system (e.g. EAWS), grants the design, the implementation and the control of safe working systems. If any, incentive systems should focus on productivity (performance is only one dimension of it) and other key performance indicators (e.g. profitability, quality, etc.) to support and achieve world class productivity levels without impairing the workers health.

\section{References}

[1] Ralph M. Barnes, Motion and Time Study Design and Measurement of Work, John Wiley \& Sons, 1980, seventh edition, p. 288

[2] Gabriele Caragnano, Performance Rating Scales, International MTM Directorate Technical Report, 2007

[3] Benjamin Niebel Andris Freivalds, Methods, Standards, and Work Design, 2005, chapter 11, Allowances

[4] EN 1005-2:2003+A1:2008. Safety of machinery - Human physical performance - Part 2: Manual handling of machinery and component parts of machinery; ISO 11228-1:2003 Ergo- 
nomics -- Manual handling -- Part 1: Lifting and carrying; EN 1005-3:2002+A1:2008. Safety of machinery - Human physical performance - Part 3: Recommended force limits for machinery operation; ISO 11228-2:2003 Ergonomics -- Manual handling -- Part 2: Pushing and pulling; EN 10054:2005+A1:2008. Safety of machinery - Human physical performance - Part 4: Evaluation of working postures and movements in relation to machinery; ISO 11226 Ergonomics -Evaluation of static working postures; ISO 11228-3: 2007 Manual handling -- Part 3: Handling of low loads at high frequency
[5] Eliezer E. Kamon, Physiological Basis for the Design of Work and Rest, Handbook of Industrial Engineering, 1982, chapter 6.4.1

[6] G. Lehman, Praktische Arbeitsphysiologie, Georg Thieme Verlag, Stuttgart, 1962, p. 68

[7] J.V.G.A. Durnin and R. Passmore, Energy, Work and Leisure, Heinemann Books, 1967, p. 74

[8] Associazione MTM Italia, IAD, EAWS Manual, 2011 